\title{
Entwicklung medizinischer Fachabteilungen - Change Management unter Kostendruck
}

Im Klinikalltag ist das Thema Change Management meistens angesiedelt im Spannungsfeld zwischen harten Rationalisierungsvorhaben, die stark am unmittelbaren betriebswirtschaftlichen Erfolg orientiert sind und wachstumsorientierten Projekten, die sich mehr auf Veränderung der Kultur oder auf Erneuerung und Innovation konzentrieren. Oft sind diese widersprüchlichen Dynamiken im Projekt zu vereinen [1]. Dies liegt u. a. darin begründet, dass kurzfristige Rationalisierungsmaßnahmen über nachhaltige Kultur- und Organisationsveränderungen in den Kliniken abgesichert werden müssen, damit ein dauerhafter Erfolg der Abteilung sichergestellt werden kann. Ist dies nicht der Fall, folgt Rationalisierung auf Rationalisierung, da das Unternehmen nicht gelernt hat, sich wachstumsorientiert auszurichten. Wie der Balanceakt zwischen konsequenten Einschnitten und neuem Wachstum gestaltet werden kann, zeigt das folgende Praxisbeispiel.

\section{Ausgangssituation}

Eine der Kliniken für Innere Medizin des Universitätsklinikums Halle (Saale) weist 2008 ein Profitcenterdefizit in Höhe von ca. $45 \%$ bezogen auf die Gesamterlöse der Abteilung aus. Neben den Verlusten charakterisierten insbesondere unklare Führungs- und Verantwortungsstrukturen, mangelnde strategische Zielrichtung sowie veraltete Prozess- und Ablaufvorgaben auf den Stationen die Ausgangslage. Die primäre Zielsetzung des Reorganisationsprojekts fokussierte auf die betriebswirtschaftlichen Ergebnisse. So sollte das Defizit innerhalb von 2,5 Jahren schrittweise abgebaut und die Abteilung in Richtung eines ausgeglichenen Ergebnisses überführt werden. Neben Rationalisierungsmaßnahmen im Rahmen des Personal- und Sachkostenbudgets sollte dies durch die Steigerung der Erlöse sowie durch eine Prozessoptimierung in den primären Leistungsbereichen erreicht werden. Die langfristige Zielsetzung bestand in der Etablierung neuer Organisationsstrukturen als Basis für eine wachstumsorientierte Weiterentwicklung der Klinik.

Personal: „You can’t shrink to success“"1 Ein wichtiger Hebel zur Realisierung betriebswirtschaftlicher Ergebnisverbesserungen liegt in Rationalisierungsmaßnahmen im Bereich des Personalbudgets. Oft werden hierbei kurzfristige Rationalisierungsziele durch Personalabbau erreicht, die jedoch langfristige Wachstumsziele blockieren. Um diesen Widerspruch aufzuheben, wurden in der Abteilung Maßnahmen zur Effizienzsteigerung

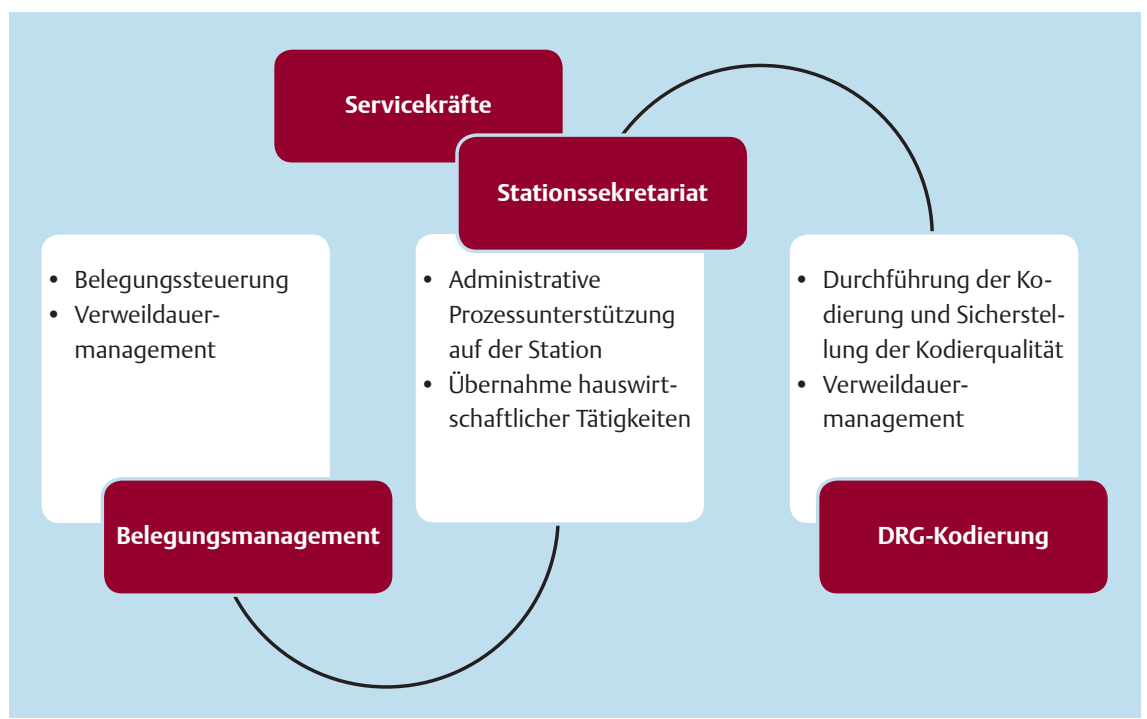

Abb. 1: Unterstützungsfunktionen

des Personaleinsatzes mit denen zur Kostenreduzierung kombiniert.

Grundlage dafür bildete ein gestuftes Personalkonzept. Entlang der behandlungsrelevanten Prozesse wurde der Einsatz von Unterstützungsfunktionen definiert. Verbindliche Ablaufstrukturen und klar geregelte Verantwortlichkeiten sicherten die bestmögliche Verzahnung der Berufsgruppen sowie die optimale Auslastung innerhalb ihrer originären Kerntätigkeiten (Abb. 1).

Zur Umsetzung der definierten Personalstruktur wurden trotz der angestrebten Rationalisierungsziele neue Stellen geschaffen (Abb. 2). Im pflegerischen Bereich trugen z. B. 2 Stellen im neu konzipierten Stationssekretariat zur Unterstützung von Arztdienst und Pflege bei. Die Einführung des Belegungsmanagements führte zu einer konsequenten Umsetzung der notwendigen Verweildauerreduzierung. Kodierfachkräfte entlasten den Arztdienst und tragen gleichzeitig zu einer Verbesserung der Abrechnungsqualität bei. Die Pflege wurde bei den hauswirtschaftlichen Tätigkeiten zusätzlich durch Servicepersonal abgelöst. Im Gegenzug übernahm die Pflege delegierbare ärztliche Tätigkeiten, wie z. B. die Applikation der Chemotherapie.

Neben dem wirtschaftlicheren Einsatz des Personals konnten zudem über eine Optimierung der Personaleinsatzplanung Kosten eingespart werden. Im ärztlichen Dienst erzielte u. a. die Abschaffung des klinikinternen Bereitschaftsdienstes einen positiven Effekt in Höhe von ca. 140000 Euro pro Jahr. Zusätzlich konnten die gewonnenen Arbeitszeiten in der Tagesroutine für die Betreuung und Behandlung von Patienten genutzt werden. Um dennoch rund um die Uhr eine zuverlässige Patientenversorgung zu wahren, wurde die Klinik stattdessen in den Bereitschaftsdienst des Departments ,Innere Medizin‘ integriert und ein Spätdienst eingerichtet.

Stationen: Unternehmen im

Unternehmen

Gemessen an Umsatz und Größe lassen sich Stationen mit kleinen mittelständi-

${ }^{1}$ Arthur Martinez, ehemals CEO von Sears 


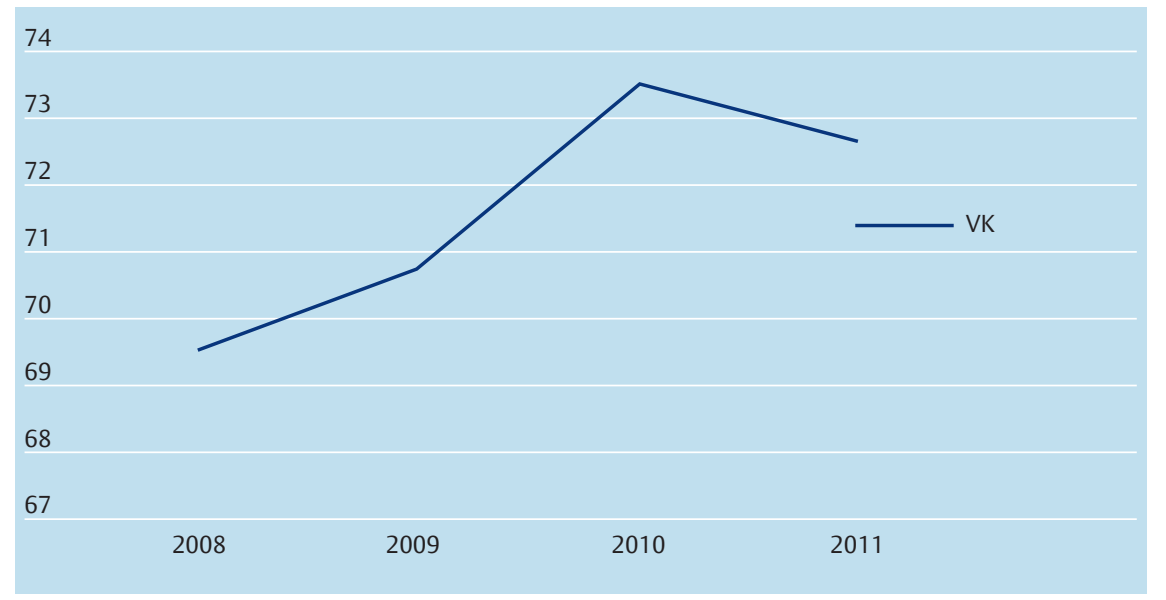

Abb. 2: Entwicklung Vollkräfte

schen Unternehmen vergleichen. Sie stellen die zentralen leistungserbringenden Einheiten mit direktem Patientenkontakt dar. Einer der entscheidenden Faktoren für die Wirtschaftlichkeit einer internistischen Abteilung ist daher die Etablierung ihrer Stationen als zentrale Verantwortungseinheit mit klaren Management- und Führungsstrukturen.

Ausgehend von dem gestuften Personalkonzept wurden die definierten Verantwortlichkeiten zwischen den Berufsgruppen im Rahmen von Stationskonzepten aufgegriffen, die der Festlegung aller relevanten Kernprozesse auf Station dienen. Bei der Definition der Führungsstrukturen wurde ein „Managementtandem“ aus stationsverantwortlichem Oberarzt und Stati-

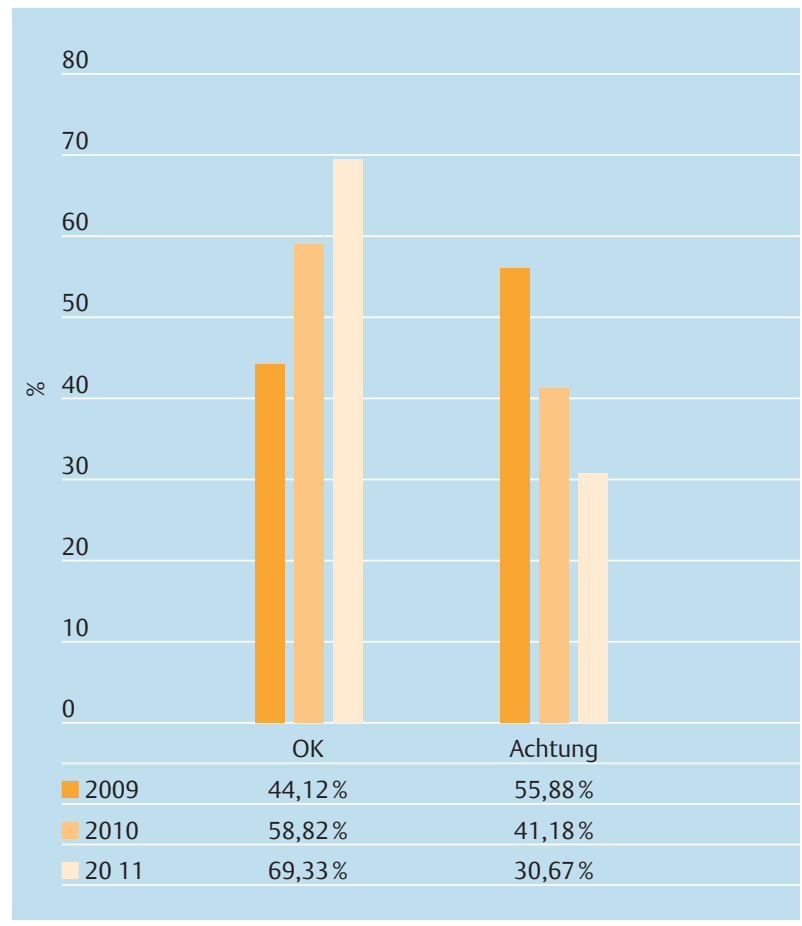

Abb. 3: Controlling Verweildauer onsleitung etabliert. Ihnen obliegt gemeinsam die Management- und Prozessverantwortung. Anhand genauer Zeitziele wurden alle wesentlichen Abläufe im und Visiten klar in ihrer Abfolge und zeitlichen Dimension festgeschrieben und dabei das Zusammenspiel der Berufsgruppen gemäß der definierten Prozesse synchronisiert. Prozessbeschreibungen für die Einarbeitung von neuen Mitarbeitern sichern die Kontinuität der beschriebenen Abläufe. Die Optimierung der Prozesse und Schnittstellen auf den Stationen mindert nicht nur unnötige Reibungsverluste in der $\mathrm{Zu}$ sammenarbeit zwischen den Berufsgruppen, sondern schärft auch die Managementverantwortung der zuständigen Mitarbeiter. Die Umsetzung der definierten Prozessziele hat zudem positive Auswirkung auf Wirtschaftlichkeitsfaktoren wie die Verweildauer (VWD) oder diagnostikund therapiefreie Belegungstage.

Um die Nachhaltigkeit der durchgeführten Struktur- und Prozessanpassungen überprüfen zu können, wurden Kennzahlen definiert. Im Rahmen des Verweildauermanagements wurden z. B. die Top 20 DRGs in ihrer Abweichung von der durchschnittlichen Verweildauer sowie im Vergleich zu Benchmarkdaten anderer Universitätskliniken erhoben und ausgewertet. Stationsalltag wie Aufnahme, Entlassung
Auf Basis der Ergebnisse wurden im ersten Schritt gezielt Maßnahmen zur Reduzierung der Verweildauer abgeleitet und erfolgreich umgesetzt.

Neben Einführung des Belegungsmanagements und zusätzlicher Verweildauerüberwachung durch die Kodierfachkraft wurden z. B. Diagnostikprofile zur schnelleren Umsetzung der Basisdiagnostik definiert oder der Prozess der Blutabnahme bei Entlasspatienten priorisiert in den Tagesablauf eingeplant. Nach erfolgreicher Etablierung der Maßnahmen wurden die Kennzahlen auf die Auswertung der Mittleren Verweildauer vereinfacht, um den Erhebungsaufwand zu reduzieren. Den positiven Effekt der durchgeführten Maßnahmen veranschaulicht die Abbildung 3.

\section{EDV: Investitionen in}

\section{Wachstumsstrukturen}

Ein Ziel der Prozessoptimierungen war es auch, die Arbeitsabläufe durch die gezielte Nutzung von EDV-Lösungen optimal zu unterstützen. Dies erforderte in einem ersten Schritt die Freigabe weiterer Investitionsmittel durch den Klinikumsvorstand. In der Diskussion wurde der durch die Investitionen langfristig realisierte Nutzen bewertet und die Mittel letztlich bewilligt.

Aufbauend auf den Planungsmöglichkeiten des KIS-eigenen BettendispositionsTools wurde ein Konzept zur Belegungsplanung und -steuerung entwickelt. Mit Einführung des Konzeptes wurde die gesamte Belegungsplanung EDV-gestützt über eine hauptverantwortliche Belegungsmanagerin abgewickelt, die als examinierte Pflegekraft über langjährige Erfahrung verfügt. Seit diesem Zeitpunkt erfolgt die Bettenbelegung ausschließlich über das Bettenmanagement und nicht mehr über einzelne Ärzte. Somit ist der Belegungsprozess von der Terminfindung über die Anmeldung von Diagnostikleistungen im Vorfeld des stationären Aufenthaltes bis hin zur Einplanung der Patienten mit definierter Verweildauer (medizinisch bestimmt und an der mittleren VWD nach DRG orientiert) klar festgelegt.

Über die Einführung der elektronischen Arztbriefschreibung wurde die im Stationskonzept verankerte Zielsetzung unterstützt, jeden Patienten bis 9:00 Uhr mit einem Arztbrief zu entlassen. Das im Krankenhausinformations-System (KIS) hinterlegte Modul mit elektronischem Diktat und Workflow zur Weiterleitung 
und Freigabe durch den Oberarzt verkürzt die Arztbrieferstellung und -laufzeit in erheblichem Maße. Zudem wurde ein Standard-Arztbrief mit z. T. definierten Textbausteinen im Modul hinterlegt. Musterarztbrief und Diktierleitfaden erleichtern insbesondere die Einarbeitung neuer Mitarbeiter. Ergänzend wurden Ressourcen im Schreibdienst optimiert.

\section{Sachkostenbereich: Kostensenkungen mit Maß}

Neben einer Neugestaltung der Ablaufstrukturen zur Optimierung der Ressourcen wurden gezielte Kostensenkungen im Sachkostenbereich durch die Zusammenarbeit mit Apotheker, Hygiene und Labormedizinern erwirkt. Durch die detaillierte Auswertung der Kosten der Apotheke sowie die Erstellung von SOPs (Standard Operating Procedures) gemeinsam mit Apotheker und Oberärzten konnte beispielsweise ein kostengünstigerer Medikamenteneinsatz erwirkt werden. Zur wirtschaftlichen Steuerung der Leistungsanforderungen wurden Labor- und Diagnostikprofile erarbeitet.

Die Maßnahmen führten in 2009 zu einer Einsparung von 300000 Euro beim medizinischen Bedarf. 2010 betrug die Einsparung gegenüber der Ausganssituation bereits 1,283 Mio. Euro. Die stationären innerbetrieblichen Leistungsverrechnungen konnten allein in 2009 um ca. 1 Mio. Euro reduziert werden.

Weitere Kostensenkungen ergaben sich durch die deutlich gesteigerte Dokumentations- und Kodierqualität, die sich insbesondere in einer geringeren Abschreibungsquote im DRG-Bereich sowie einer gesteigerten Abrechnung von z. B. Zusatzentgelten niederschlug.

Neben einem stringenten Kostencontrolling war für die Realisierung dieses Einsparungspotenzials entscheidend, dass bei allen Beteiligten ein Bewusstsein für die monetären Auswirkungen medizinischen Handelns geweckt werden konnte. Die Behandlungsqualität und die Patientenzufriedenheit haben im Rahmen der durchgeführten Maßnahmen sogar zugenommen. Dies liegt u. a. daran, dass die Rationalisierungs- und Kosteneinsparmaßnahmen abgestimmt innerhalb des Gesamtkonzeptes durchgeführt wurden.

\section{Neue Strukturen:}

\section{Basis für Leistungssteigerung}

Dass die Etablierung neuer Organisationsstrukturen als Basis für eine stabili- sierte Weiterentwicklung der Abteilung gelungen ist, zeigen die Leistungszahlen. Trotz der signifikanten Kosteneinsparungen konnten die Leistungen gehalten werden. Im Bereich der DRG-Erlöse konnten 2009 und 2010 sogar in Summe eine Erlössteigerung von über 1 Mio. Euro gegenüber der Ausgangssituation erzielt werden. Ein zentraler Erfolgsfaktor hierbei war die Einführung von persönlichen Besuchen der Zuweiser durch die verantwortlichen Oberärzte und die Verbesserung der ambulant-stationären Schnittstelle.

Wie das Praxisbeispiel verdeutlicht, funktioniert eine ausbalancierte Strategie zwischen konsequenten Einschnitten und Wachstumsorientierung und macht Reorganisationsprojekte auch unter Kostendruck zu einem Erfolgsmodell.

\section{Literatur}

1. Heitger B, Doujak A (Hrsg.): Harte Schnitte - Neues Wachstum. Die Logik der Gefühle und die Macht der Zahlen im Change Management. Das Konzept der unbalanced Transformation. Wirtschaftsverlag bei Ueberreiter, Frankfurt 2002.

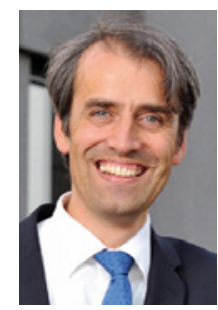

Korrespondenz Stefan Ruhl Ruhl Consulting AC Harrlachweg 1 68163 Mannheim Tel.: $0621328850-0$ Fax: $0621328850-50$ E-Mail: stefan.ruhl@ ruhl-consulting.de

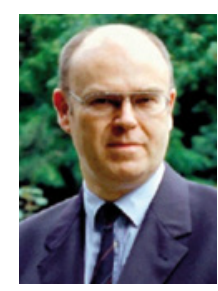

Privat-Dozent Dr. Thomas Klöss Ärztlicher Direktor und Vorsitzender des Vorstandes Universitätsklinikum Halle (Saale) Ernst-Grube-Str. 40 06060 Halle (Saale) Tel.: 0345-5574480 E-Mail: adirektor@uk-halle.de 